\title{
Phosphorylated ezrin is located in the nucleus of the osteosarcoma cell
}

\author{
Claudio Di Cristofano, Martina Leopizzi, Antonella Miraglia, Barbara Sardella, \\ Valentina Moretti, Alessandro Ferrara, Vincenzo Petrozza and Carlo Della Rocca \\ Department of Experimental Medicine, Sapienza University of Rome, Polo Pontino, I.C.O.T, Latina, Italy
}

\begin{abstract}
The survival of osteosarcoma patients is connected to metastasis. The ezrin expression is associated with the development of metastasis and poor outcome in osteosarcoma. Ezrin is present in the cytoplasm and after phosphorylation assumes an active form and links F-actin to the cell membrane. This study evaluated ezrin and phosphorylated ezrin at site Tyr354 and Thr567 expression and its subcellular localization in osteosarcoma. We studied 50 osteosarcoma patients (mean follow-up 9.8 years). Ezrin expression was assessed using immunohistochemical and immunofluorescence analysis on tissue microarray and cultured cells of human osteosarcoma 143B. The western blot analysis was carried out on cultured cells. The majority of osteosarcomas, showing cytoplasmic positivity for ezrin, phosphorylated and unphosphorylated, were associated with membranous and nuclear positivity for phosphorylated ezrin Thr567 and phosphorylated ezrin Tyr354, respectively. Ezrin expression was associated with high-grade osteosarcoma $(P=0.04)$, with metastasis $(P=0.04)$ and with tumors that developed metastasis $(P=0.04)$; phosphorylated ezrin Thr567 expression was present mostly in tumors with metastasis $(P=0.01)$ and in osteosarcomas that did not develop metastasis $(P=0.002)$. The osteosarcoma patients with ezrin expression have a short survival. The cytoplasmic ezrin expression in osteosarcoma matches its role of membrane-cytoskeleton linker protein. The subcellular trafficking of ezrin is not blocked and it is linked to ezrin phosphorylation, also in cancer. The phosphorylated ezrin Tyr354 nuclear localization suggests its possible role as a nuclear factor in osteosarcoma. The phosphorylated ezrin Thr567 phosphorylation may not be necessary in osteosarcoma metastatic progression but it was modulated. The ezrin expression is associated with more aggressive osteosarcomas and with metastasis.
\end{abstract}

Modern Pathology (2010) 23, 1012-1020; doi:10.1038/modpathol.2010.77; published online 26 March 2010

Keywords: ezrin; phosphorylated ezrin Thr567; phosphorylated ezrin Tyr354; osteosarcoma; tissue microarray; subcellular localization

Osteosarcoma is the most common primary bone tumor in pediatric-age patients, in which osteoid or bone is produced directly by tumor cells. ${ }^{1}$

Osteosarcoma includes several different clinicalpathological entities, differing in clinical, radiological, and histological features. Some of these entities are of prognostic significance, indicating a low or high grade of malignancy. ${ }^{1}$

The treatment of osteosarcoma is based on a combined approach of primary antiblastic chemotherapy and tumor surgery, followed by adjuvant chemotherapy. ${ }^{2}$

Correspondence: Dr C Di Cristofano, MD, Department of Experimental Medicine, Sapienza University of Rome, Polo Pontino, I.C.O.T, Via F. Faggiana, 43, Latina 00143, Italy.

E-mail: claudio.dicristofano@uniroma1.it

Received 14 October 2009; revised and accepted 7 December 2009; published online 26 March 2010
The survival of patients affected by osteosarcoma is connected to metastasis. ${ }^{3}$ Patients who present a metastatic disease on diagnosis or who develop metastatic progression usually have a worse prognosis. Metastatic tumors are often nonrespondent or only partially responsive to current therapeutic strategies and are the primary cause of cancerrelated mortality. ${ }^{4}$

The identification of molecular events linked to the metastatic process is crucial for understanding tumor dissemination and for the development of novel therapy.

Currently, some studies have tried to correlate the up- or downregulation of molecular pathway components with better prognosis or with metastasis. ${ }^{5}$

Gene expression and proteomic studies have identified the possible role of ezrin in metastasis. ${ }^{6,7}$

The identification of ezrin expression is a necessary component in osteosarcoma metastasis and it 
makes the study of this protein crucial in understanding the molecular pathways involved in metastatic diffusion. ${ }^{8}$

Ezrin, the product of the Vil2 gene, is present in the cytoplasm in an inactive form but after threonine and tyrosine phosphorylation, ezrin assumes an active form, moving to the cell membrane and tethering $\mathrm{F}$-actin to the cell membrane. ${ }^{9}$

Ezrin has been associated with the formation of specialized cell surface domains and assists in the placement of molecules within these structures. ${ }^{10}$ It is known to be a component of cell surface structures that are involved in cell adhesion to the extracellular matrix, as well as in cell-cell interactions, receptor tyrosine kinase signaling, signal transduction through Rho GTPase and interactions with the Akt-mediated cellular apoptotic machinery. ${ }^{11,12}$

Phosphorylation of ezrin promotes morphogenesis of apical microvilli and regulates E-cadherindependent adherent junction assembly. ${ }^{13-15}$ Ezrin directly interacts with signaling enzymes such as phosphoinositide 3-kinase (PI3-kinase), and the phosphorylated tyrosine 353 (Tyr-353) residue of ezrin is regulated through the PI3-kinase/Akt pathway. ${ }^{11}$ Ezrin is also preferentially degraded and resynthesized after oxidative stress ${ }^{16}$ and the phosphorylation at threonine 567 (Thr-567) depends on the p38 MAP-kinase activity. ${ }^{17}$ However, the detailed signal transduction pathways of phosphorylated ezrin remain unclear.

Ezrin has been implicated in many roles in the metastatic process, for example, as a conduit for signals between metastasis-associated cell surface molecules and signal transduction components. ${ }^{18}$

Ezrin as a key component of pediatric tumor metastasis adds an important link to the molecular chain connecting metastasis-associated cell surface proteins to the signal transduction network and, ultimately, to the nucleus. ${ }^{19}$

Transfection of full-length Vil2 constructs in lowmetastatic cell lines dramatically increase the ability of these cell lines to form macroscopic pulmonary lesions in experimental metastasis assays, demonstrating that ezrin overexpression is sufficient to confer metastatic capacity. ${ }^{20}$

In a mouse model of osteosarcoma, ezrin was necessary for metastasis, and in dog tumors, a high expression of ezrin was associated with the early development of metastasis. ${ }^{8}$ A relationship was also shown between high expressions of ezrin and poor outcome in 19 pediatric osteosarcoma patients. ${ }^{8}$

More importantly, using dominant-negative mutants, antisense RNA or RNA interference, both groups demonstrated that ezrin overexpression is not only sufficient for metastatic progression, but it is also necessary, at least in these experimental systems. ${ }^{8}$

Suppression of ezrin protein expression in osteosarcomas by antisense transfection or stable expression of short hairpin RNA, or the disruption of ezrin function by transfection of a dominant-negative ezrin significantly reduced the metastatic behavior in both mouse models and was associated with decreased Akt and mitogen-activated protein kinase (MAPK) activity. ${ }^{20}$ The mechanisms of ezrin-related metastatic behavior are linked to an Akt-dependent mammalian target of rapamycin (mTOR) ${ }^{21}$ However, the specific mechanism or mechanisms by which ezrin mediates the metastatic process remain to be clarified.

On the basis of the relevant role of ezrin in tumor progression and metastasis, we decided to investigate the ezrin protein expression in a series of osteosarcomas, focusing on the immunohistochemical subcellular distribution of this protein in its active (phosphorylated) and inactive (unphosphorylated) forms. The data obtained were compared with the clinical-pathological characteristics of the tumors. The hypothesis was that the movement of the ezrin was tied up to its state of phosphorylation, also in tumors, and that ezrin expression and subcellular localization were involved in osteosarcoma metastatic progression.

\section{Materials and methods}

\section{Population Study}

The population of this study included 58 formalinfixed, paraffin-embedded blocks of surgical osteosarcoma specimens obtained from the files of the Department of Experimental Medicine, Sapienza University of Rome, Polo Pontino, I.C.O.T, Latina, Italy.

The osteosarcoma specimens included 22 biopsies, 31 surgical resections, and 5 metastatic lesions, and they corresponded to 50 patients.

We had the following series available:

- 23 patients with only surgical resection specimens;

- 17 patients with only surgical biopsy specimens;

- 5 patients with surgical biopsy and resection specimens;

- 3 patients with surgical resection and metastasis specimens;

- 2 patients with only surgical metastasis specimens;

Twenty-three patients with osteosarcoma were clinically followed up with mean follow-up of 9.8 years (range 1-18 years) with a 56\% overall survival.

The WHO 2008 classification of bone tumors was used for classifying the bone lesions (WHO 2008). The osteosarcoma sites and types are described in Table 1.

The osteosarcoma grade was available in 48 patients on 31 resection and 17 biopsy specimens and were 40 of $48(83 \%)$ and 8 of $48(17 \%)$ high grade and low grade, respectively.

\section{Tissue Microarray Construction}

Core tissue biopsies of $1 \mathrm{~mm}$ in diameter were taken from representative regions of each paraffin- 
Table 1 Clinical-pathological data of primary osteosarcomas

\begin{tabular}{|c|c|c|}
\hline \multicolumn{3}{|l|}{ Age } \\
\hline $13 / 48$ & $-27 \%$ & $\leq 18$ years \\
\hline $35 / 48$ & $-73 \%$ & $\geq 18$ years \\
\hline \multicolumn{3}{|c|}{ Type of osteosarcoma } \\
\hline $37 / 48$ & $77 \%$ & Conventional osteosarcoma \\
\hline $5 / 37$ & $14 \%$ & Osteoblastic osteosarcoma \\
\hline $4 / 37$ & $11 \%$ & Chondroblastic osteosarcoma \\
\hline $1 / 37$ & $3 \%$ & Fibroblastic osteosarcoma \\
\hline $5 / 48$ & $1 \%$ & Parosteal osteosarcoma \\
\hline $4 / 48$ & $8 \%$ & Periosteal osteosarcoma \\
\hline $2 / 48$ & $4 \%$ & Telangiectatic osteosarcoma \\
\hline \multicolumn{3}{|c|}{ Site of osteosarcoma } \\
\hline $26 / 48$ & $54 \%$ & Femur \\
\hline $10 / 48$ & $21 \%$ & Tibia (flute) \\
\hline $4 / 48$ & $8 \%$ & Humerus \\
\hline $2 / 48$ & $4 \%$ & Fibula (buckle) \\
\hline $2 / 48$ & $4 \%$ & Jaw \\
\hline $2 / 48$ & $4 \%$ & Mandible \\
\hline $1 / 48$ & $2 \%$ & Lumbar vertebra \\
\hline $1 / 48$ & $2 \%$ & Ulna \\
\hline \multicolumn{3}{|l|}{ Stadio } \\
\hline I B & 6 & \\
\hline II A & 7 & \\
\hline II B & 35 & \\
\hline
\end{tabular}

embedded tumors (donor block) and arrayed into a new recipient paraffin block $(45 \mathrm{~mm} \times 20 \mathrm{~mm})$ using the ATA-100 Chemicon International System. To minimize the influence of tumor and tissue heterogeneity, three different core biopsies for each donor block were retrieved. Each array contained 50 tissue cylinders; there were 30 malignant tissue tumors corresponding to 10 patients and 20 normal tissue controls.

\section{Cell Culture}

Human osteosarcoma cell lines (143B) were grown under subconfluent or confluent conditions in medium, at $37^{\circ} \mathrm{C}$ with $5 \%$ of $\mathrm{CO}_{2}$. Cells were cultured in Dulbecco's minimum essential medium (Sigma) supplemented with $10 \%$ fetal bovine serum (GIBCO) with penicillin $(100 \mu \mathrm{g} / \mathrm{ml})$, streptomycin $(100 \mathrm{U} / \mathrm{ml})$ and sodium pyruvate.

Cells were cultured on cover glass $(12 \mathrm{~mm} \varnothing)$ for immunohistochemical and immunofluorescence analysis and were fixed with $3 \%$ paraformaldehyde/phosphatase-buffered saline and incubated with primary antibodies.

\section{Protein Expression Analysis (Immunohistochemical and Immunofluorescence)}

Immunohistochemistry

Immunohistochemical detection of ezrin unphosphorylated (ezrin), phosphorylated ezrin at Tyr354 level (p-ezrinTyr354), and phosphorylated ezrin at Thr567 level (p-ezrinThr567) expression was performed with standard streptavidin-biotin- peroxidase on cell-culture confluent and subconfluent and tissue microarray sections.

Histological sections $(2 \mu \mathrm{m})$ were deparaffinized and rehydrated in graded ethanol. Endogenous peroxidase activity was blocked by $3 \%$ hydrogen peroxide for $10 \mathrm{~min}$. Antigen retrieval was performed in $10 \mathrm{mM}$ sodium citrate buffer ( $\mathrm{pH}$ 6.0) for $15 \mathrm{~min}$. The tissue microarray sections and cell culture were then incubated with mouse IgG monoclonal antibody anti-ezrin (clone 3C12-Santa Cruz Biotechnology) 1:150; rabbit IgG polyclonal antibody anti-phosphorylated ezrin (Tyr 354-Santa Cruz Biotechnology) 1:50; and rabbit IgG polyclonal antibody anti-phosphorylated ERM (Ezrin (Thr567)/ Radixin (Thr564)/Moesin (Thr558) (Cell Signalling Technology) 1:50, at room temperature for $1 \mathrm{~h}$. After incubation, specimens were washed with PBSTween buffer and with the secondary biotinylated antibody and subsequently with the streptavidinbiotin-peroxidase (DAKO LSAB Kit peroxidase; DAKO, Carpinteria, CA, USA). The samples were then washed with PBS-Tween buffer and incubated with freshly prepared DAB + substrate-chromogen buffer at room temperature. After gently rinsing with $\mathrm{dH}_{2} \mathrm{O}$, slides were counterstained with hematoxylin and mounted with permanent mounting media. Both positive and negative controls were used in each experiment.

\section{Immunofluorescence}

Immunofluorescence detection of ezrin, p-ezrinTyr354, and p-ezrinThr567 expression was performed on cell-culture confluent and subconfluent and tissue microarray sections.

Immunofluorescence was performed on 2 - $\mu$-thick tissue microarray sections and on cellular cultures. Sections and cellular cultures were incubated with primary antibody as described before and then with fluoresceinated secondary antibody. The anti-mouse Alexa Fluor 488 antibody or anti-rabbit Alexa Fluor 488 antibody (1:300) was applied for $2 \mathrm{~h}$ at $37^{\circ} \mathrm{C}$ after three washes in $0.1 \%$ Tween in PBS for 10 min each. Slides were counterstained with propidium iodide and mounted in glycerol/buffer on a glass slide after extensive washing with PBS.

\section{Light and Confocal Microscope}

The reaction was examined by light microscope (Leika) for immunohistochemical analysis and by confocal laser microscope (Nikon TE2000) for immunofluorescence analysis.

Placental tissues, breast, and stomach cancer tissues were used as immunohistochemical and immunofluorescence positive controls for ezrin and p-ezrin, respectively. For negative controls, the primary antibody was omitted.

Only cases in which all the three tissue microarray tumor samples could be evaluated were 
considered in the study. Cases were grouped into two classes: negative cases, when the cases showed some immunohistochemical expression of AB in all three samples, and positive cases, in which the tumor cells showed in at least $1 \%$ of positive cells in all three samples.

Membranous, cytoplasmic, and nuclear expressions were also independently assessed.

\section{Western Blot Analysis and Subcellular Fractionation}

Only subconfluent cultured cells were lysed in RIPA buffer (150 mM NaCl, 50 mM Tris, pH 8, 0.1\% SDS, $0.25 \%$ deoxycholate, $1 \%$ NP-40) with protease inhibitor cocktail (Roche Diagnostics, Indianapolis, IN, USA) and $1 \mathrm{mM}$ calyculin A (Alexis Biochemicals, Lausen, Switzerland) for whole-cell protein. For nuclear extraction, the Active Motif Nuclear Extract kit (Active Motif, Carlsbad, CA, USA) was used following the manufacturer's instructions. The proteins were frozen at $-70^{\circ} \mathrm{C}$ until used. The concentrations of protein were measured by the Bradford method. Western blot analysis was carried out after electrophoresis in SDS/10\% polyacrylamide gels, and the membrane was incubated only with ezrin (1:2000 dilution) or p-ezrinTyr354 (1:200 dilution) antibodies for $2 \mathrm{~h}$ at room temperature, then incubated with horseradish peroxidase-conjugated goat anti-mouse IgG (1:10000) for $1 \mathrm{~h}$ at room temperature and proteins were detected using enhanced chemiluminescence (Amersham).

\section{Statistical Analysis}

The two-tailed Fisher's exact test was used to analyze the correlation of the different variables. The probability of survival was estimated by the Kaplan-Meier method, with the log-rank test used to estimate differences among levels of the analyzed variables.

\section{Results}

\section{Immunohistochemical Protein Expression and Localization}

Tissue microarray samples

Positive osteosarcoma immunoreactivity for ezrin was detected in 43 of 50 (86\%) cases, p-ezrinTyr354 was positive in 43 of $50(86 \%)$ cases, and 44 of 50 $(88 \%)$ cases were positive for p-ezrinThr567. All ezrin-positive osteosarcoma cases only showed positive to the cytoplasmic level (Figures 1a and b).

The p-ezrinTyr354 in osteosarcomas showed a cytoplasmic stain in 36 of 50 (72\%) and nuclear stain in 25 of 50 (50\%). The osteosarcoma-positive cases for p-ezrinTyr354 showed both cytoplasmic and nuclear stains in 18 of 43 (42\%) (Figures 1c and d), whereas 18 of $43(42 \%)$ and 7 of $43(16 \%)$ cases showed only cytoplasmic and nuclear positivity, respectively.

The p-ezrinThr567-positive osteosarcoma showed only cytoplasmic and membranous positivity in 38 of $44(86 \%)$ and 6 of $44(14 \%)$ samples, respectively (Figures 1e and f). All cases with membranous staining also showed cytoplasmic positivity (Table 2).

The ezrin positivity was associated with p-ezrinTyr354 expression $(P=0.01)$, but if we consider the subcellular localization, only nuclear expression was associated with ezrin expression $(P=0.02)$. Ezrin expression was associated only with p-ezrinThr567 membranous positivity $(P=0.004)$. There was a statistical association of p-ezrinTyr354 and p-ezrinThr567 cytoplasmic expression $(P=$ 0.004).

\section{Cell-Culture Samples}

The immunohistochemistry was performed on confluent and subconfluent osteosarcoma culture cells.

In the confluent cell culture, we found ezrin localized at the cytoplasmic level; p-ezrinThr567 (Figure 2a) and Tyr354 (Figure 2b) localized at both cytoplasmic and membranous levels.

In the subconfluent cell culture we found ezrin localized at the cytoplasmic level; p-ezrinThr567 localized at the cytoplasmic and membranous levels (Figure 2a, lower-right panel); and p-ezrinTyr354 localized in cytoplasmic and nucleus levels (Figure 2b, lower-right panel).

\section{Immunofluorescence Protein Expression and Localization in Tissue Microarray and Cell Culture}

Tissue microarray samples

Immunofluorescence on tissue microarray sections confirmed the localization of ezrin, p-ezrinTyr354, and p-ezrinThr567 shown by immunohistochemistry. In fact, we found ezrin, p-ezrinThr567, and p-ezrinTyr354 expression localized at the cytoplasmic level only, cytoplasmic/membranous, and cytoplasmic/nuclear levels, respectively.

\section{Cell-culture samples}

The immunofluorescence was performed on confluent and subconfluent osteosarcoma culture cells, with the same results that were found in the immunohistochemical analysis.

In the confluent cell culture, we found ezrin localized at the cytoplasmic level; p-ezrinThr567 and Tyr354 localized at both cytoplasmic and membranous levels.

In the subconfluent cell culture we found ezrin localized at the cytoplasmic level; p-ezrinThr567 localized at the cytoplasmic and membranous levels (Figure 2c); p-ezrinTyr354 localized in cytoplasmic and nucleus levels (Figure 2d). 

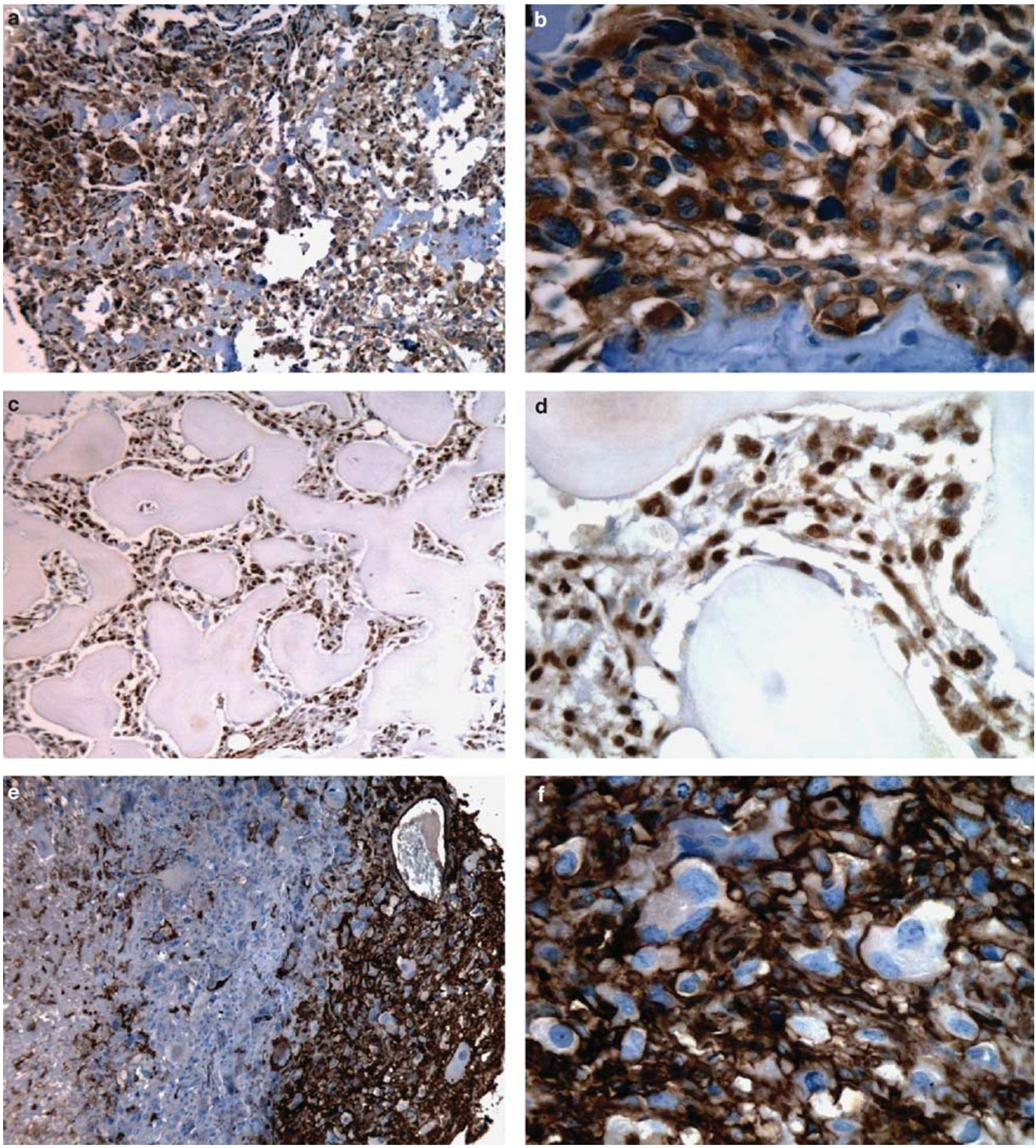

Figure 1 Immunohistochemical ezrin phosphorylated and unphosphorylated localization in osteosarcomas. (a) Unphosphorylated ezrin expression localized at cytoplasmic levels $(\times 200)$; (b) unphosphorylated ezrin expression localized at cytoplasmic levels $(\mathbf{b} \times 400)$; $(\mathbf{c})$ p-ezrinTyr354 expression localized in cytoplasmic and nucleus levels $(\times 200)$; (d) p-ezrinTyr354 expression localized in cytoplasmic and nucleus levels $(\times 400)$; $(\mathbf{e})$ p-ezrinThr567 expression localized at cytoplasmic and membranous levels $(\times 200)$. (f) p-ezrinThr567 expression localized at cytoplasmic and membranous levels $(\times 400)$

Table 2 Immunohistochemical results of ezrin phosphorylated and unphosphorylated expression in osteosarcomas

\section{Erzin}

p-EzrinTyr354

p-EzrinThr567
$36 / 50(72 \%)$

$43 / 50(86 \%)$

$25 / 50(50 \%)$

$44 / 50(88 \%)$
$6 / 50(12 \%)$ 

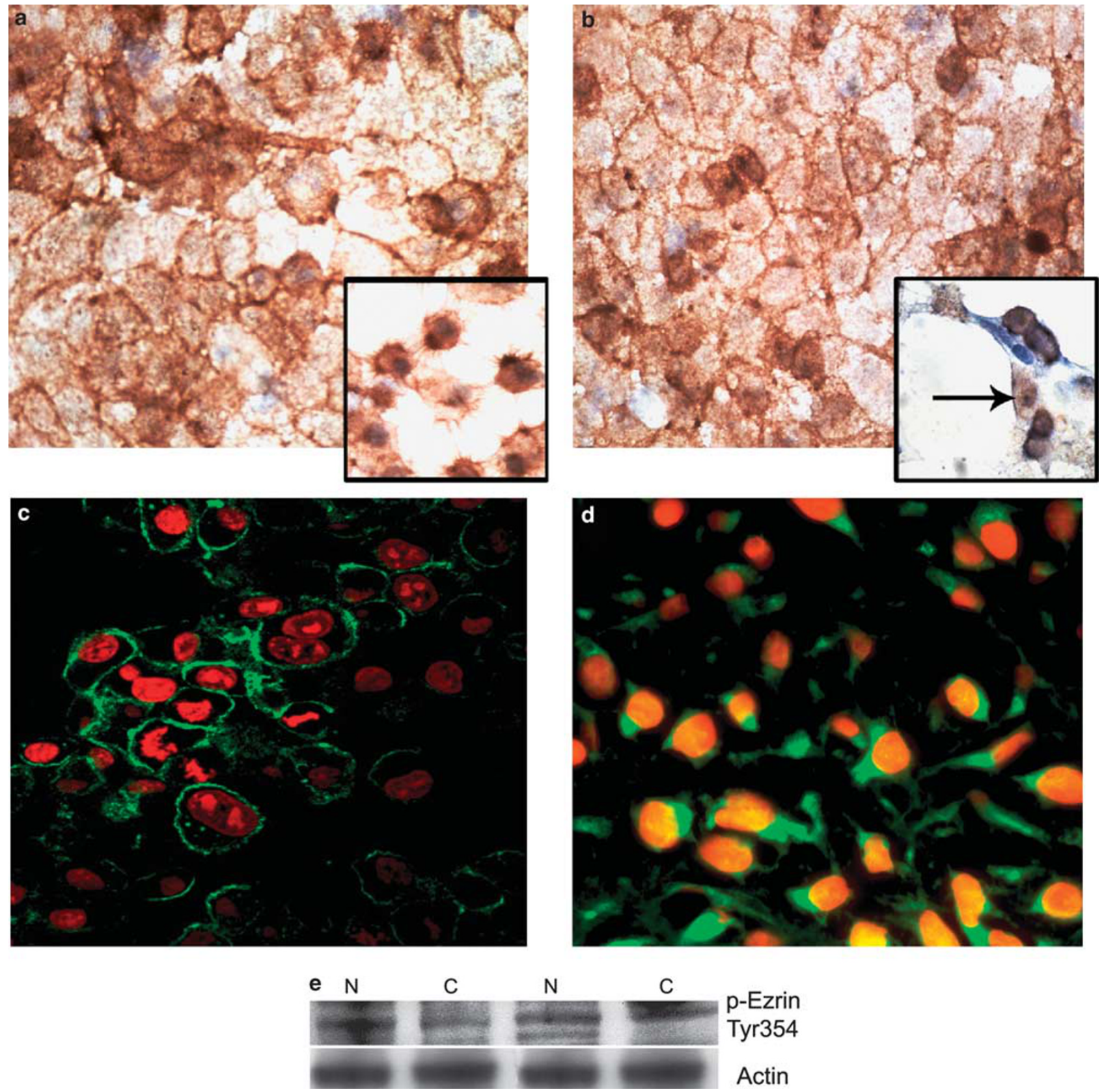

Actin

Figure 2 Immunohistochemical, immunofluorescence, and subcellular fractionation western blot analysis of p-ezrinThr567 and p-ezrinTyr354 in osteosarcoma cell culture. (a) Immunohistochemistry: p-ezrinThr567 localized at cytoplasmic and membranous levels; (a lower-right panel) immunohistochemistry: p-ezrinThr567 localized at cytoplasmic and membranous levels in subconfluent cell culture; (b) immunohistochemistry: p-ezrinTyr354 localized in cytoplasmic and nucleus levels; (b lower-right panel) immunohistochemistry: p-ezrinTyr354 localized in cytoplasmic and nucleus levels in subconfluent cell culture; (c) immunofluorescence: p-ezrinThr567 localized at cytoplasmic and membranous levels; (d) immunofluorescence: p-ezrinTyr354 localized in cytoplasmic and nucleus levels; (e) western blot analysis shows the p-ezrinTyr354 localized in cytoplasmic and nucleus levels.

\section{Western Blot Analysis and Subcellular Fractionation in Cell Culture}

The western blot analysis was carried out only on the subconfluent osteosarcoma culture cell to confirm the nuclear localization of p-ezrinTyr354. In fact, we found p-ezrinTyr354 localized in the cytoplasmic and nucleus levels (Figure 2e).

\section{Clinical-Pathological and Protein Expression Correlation}

There were not any significant differences in ezrin expression, phosphorylated and unphosphorylated, between histological osteosarcoma subtypes. However, all chondroid components of chondroblastic osteosarcoma and parosteal osteosarcoma showed 


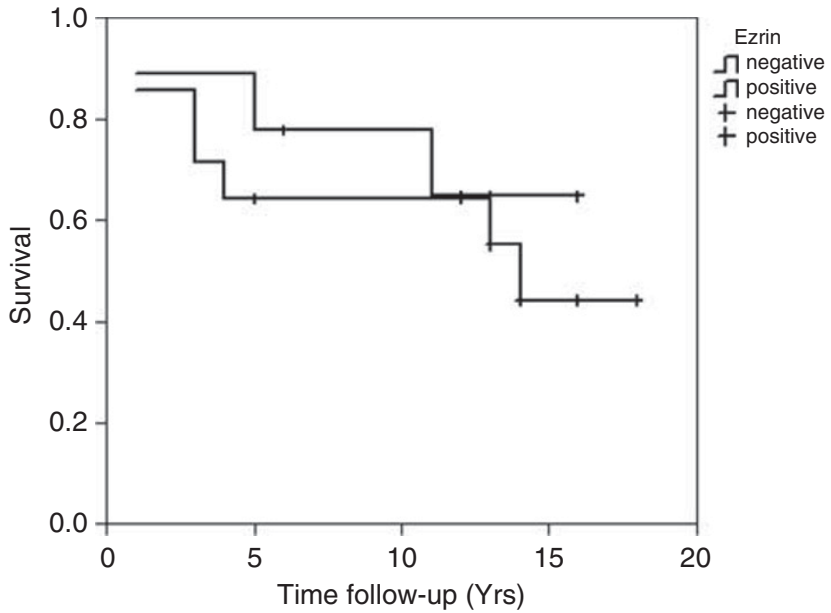

Figure 3 Tumor-specific survival analysis of ezrin unphosphorylated expression.

positive immunoreactivity for ezrin, p-ezrinTyr354, and p-ezrinThr567 at the cytoplasmic level and p-Tyr354 with nuclear localizations.

Only ezrin expression was statistically associated with high-grade osteosarcoma $(P=0.04)$. We found that ezrin expressions were associated with metastatic lesions with respect to primary tumors $(P=0.04)$. Ezrin expression was present mostly in primary tumors that developed metastasis $(P=0.04)$. Although the cytoplasmic and membranous p-ezrinThr567 expression was present mostly in primary tumors with respect to metastatic lesions $(P=0.01)$, we have found this expression in association with primary tumors that did not develop metastasis $(P=0.002)$.

\section{Tumor-Specific Survival Analysis}

No statistical relation was found between the outcome in patients with osteosarcoma and ezrin expression, phosphorylated and unphosphorylated. However, the overall survival was 50, 59, and 64\% in ezrin, Tyr347, and Thr567 phosphorylated ezrinpositive cases, respectively, and the patients with ezrin expression in primary tumors had a worse prognosis for survival (Figure 3).

\section{Discussion}

ERM proteins (Ezrin, Radixin, and Moesin) are involved in tumor development and progression and regulate cellular activities, such as survival, adhesion, and migration/invasion by organizing membrane-cytoskeleton-associated complexes. ${ }^{10,22}$ In osteosarcoma, it has been reported that ezrin is necessary for metastasis in a mouse model and that high ezrin expression in dog tumors is associated with early metastases development. ${ }^{8}$ Furthermore, a relation between high ezrin expression and poor outcome in 19 pediatric osteosarcoma patients has been described. ${ }^{2}$

This study tested different forms of ezrin and its subcellular localization in osteosarcomas, with regard to the different functional states of this protein: active phosphorylated form (p-ezrinTyr354; p-ezrinThr567) and inactive unphosphorylated form (ezrin). The data obtained were compared with the clinical-pathological characteristics of tumors.

The ezrin function link to mobility and cellular adhesion seems to depend on the specific ezrin residual Tyr354 phosphorylation through the modulation of the same one in cellular tubulogenesis. ${ }^{23}$

The phosphorylation of residual Thr567 in ezrin opens the protein to accessible domains for binding to membrane and F-actin and it is crucial for the membrane-cytoskeleton anchorage function. ${ }^{24}$

Studies carried out on normal cell cultures have underlined that ezrin is located in the microvillus, filopodis, and the membrane task, and it therefore contributes to the organization of dynamic cell structures implicated in the cellular motility. ${ }^{25}$

The cellular redistribution of ezrin from the cytoplasm to the microvillus and task membrane has been shown in many cancer cell lines treated with growth factors. ${ }^{26}$ Besides, the ezrin localization in these structures has been associated with an improvement of cell invasion, migration, and proliferation..$^{22}$

The ezrin abnormal location could cause a loss of normal anchorage to the cellular membrane of adhesion molecules or receptors or promote the activation of intracellular oncogenetic signals. ${ }^{27}$ Moreover, the loss of the apical location of ezrin could interfere with the maintenance of cell polarity and could promote the acquisition of an aggressive or metastatic phenotype in cancer. ${ }^{26}$

The hypothesis was that the movement of ezrin was tied up to its state of phosphorylation, also in tumors. In this study, we used Abs anti-ezrin unphosphorylated, anti-ezrin phosphorylated at Tyr354 site, and anti-ERM (Ezrin (Thr567)/Radixin (Thr564)/Moesin (Thr558), Cell Signaling Technology) $\mathrm{Ab}$, because at present, an $\mathrm{Ab}$ that is specific for phosphorylated ezrin at Thr567 site does not exist.

In our osteosarcoma tissue microarray samples, ezrin showed cytoplasmic immunostaining only, whereas p-ezrinTyr354 also showed nuclear localization and p-ezrinThr567 rarely showed membranous expression associated with cytoplasmic localization.

These results show that phosphorylated ezrin transmigrates to the nucleus or in the membranes in osteosarcomas and cytoplasm-nucleus and cytoplasm-membrane movement is not blocked in neoplastic cells and it is linked to the site of ezrin phosphorylation, also in tumors.

The different protein subcellular localizations could be in relation to different activities developed by this protein, also in tumors. The cytoplasmic ezrin and cytoplasmic and membranous 
p-ezrinThr567 expression could confirm the ezrin role, also in osteosarcomas, of the bond protein between F-actin and the cell membrane.

The presence of ezrin in the nucleus has been noted in vitro ${ }^{19}$ and in vivo. ${ }^{26}$ We have demonstrated for the first time the nuclear localization of the p-ezrinThr354 in osteosarcomas using immunohistochemical analysis. We have confirmed this data by immunofluorescence in tissue microarray (data not shown) sections and by immunofluorescence and subcellular fractionation western blot analysis in subconfluent osteosarcoma-cultured cells.

The nuclear localization of p-ezrinThr354 active forms in osteosarcomas suggests a possible role of the nuclear transcription factor in cancer. Further studies are necessary to confirm this hypothesis.

Kaul et $a l^{28}$ found a nuclear localization of the $\mathrm{N}$-terminus ezrin fragment of $55 \mathrm{kDa}$, where the Tyr 354 site of phosphorylation is located. He postulated that this ezrin nuclear expression could be involved in communication between the cytoskeleton and nucleus. $^{28}$

We have found different localizations of the p-ezrinTyr354 expression in immunofluorescence tests on confluent and subconfluent cell cultures. In fact, in the confluent cell culture the p-ezrinTyr354 expression was cytoplasmic and membranous, and in the subconfluent study it was cytoplasmic and nuclear. The different ezrin localization among the confluence and subconfluence cell culture has already been described. ${ }^{19}$ The authors showed that the relative nucleocytoplasmic distribution of cytoskeleton-associated proteins would therefore be cell type and cell context dependent. There is an increase in the number of cells expressing nuclear ezrin when there is a mutant form of ezrin, which lacks the actin-binding domain. ${ }^{19}$ In our study, we do not know the effective state of the whole protein or the Vil2 gene in our tumor samples.

In our study, only ezrin unphosphorylated cytoplasmic expression was associated with high-grade osteosarcoma and with metastatic lesions with respect to primary tumors. Ezrin expression was present mostly in primary tumors that developed metastasis. This result shows a correlation among the cytoplasmic ezrin expression, osteosarcoma aggressiveness, and metastatic progression.

Although the cytoplasmic and membranous p-ezrinThr567 expression was associated with primary tumors with respect to metastatic lesions, we found that this expression was associated with primary tumors that did not develop metastasis. Ren et $a l^{29}$ has recently shown that the Thr 567phosphorylated ezrin expression decreased in osteosarcoma metastatic lesions.

Our findings are in agreement with the hypothesis that ezrin phosphorylation at Thr567 level may not be necessary for osteosarcoma metastasis but it is modulated during the metastatic progression.

We found an interesting association between ezrin expression and osteosarcoma type as a chondroblas- tic subtype and a chondroid component of parosteal osteosarcoma. These data have already been found recently in other papers, in which the authors emphasize the role of ezrin in the differential diagnosis among chondroid and osteoid tumors. ${ }^{30}$ It is possible that ezrin may be involved in chondroid differentiation of osteosarcoma.

We observed that immunohistochemical detection of ezrin is not statistically associated with poor prognosis as reported for other cancer types, such as uveal malignant melanoma, ${ }^{31}$ high-grade soft tissue tumors, $^{32}$ or recently found in osteosarcomas. ${ }^{2}$ However, the patients with ezrin expression in primary tumors have a worse prognosis for survival.

These findings support the relevance of ezrin in the metastatic process in osteosarcoma. In our study, the possible role in the metastatic process of ezrin subcellular movement has not emerged, but, nevertheless, we have shown that it is not blocked and it is linked to the site of ezrin phosphorylation, also in tumors. It is possible that ezrin phosphorylation may not be necessary for the metastatic process but it is modulated.

Considering the rapid development of targeted molecular therapies, a more detailed knowledge of the specific behavior of metastasis-related proteins and their activation or activated pathways is required.

A comparison between immunohistochemical and molecular data in the group of tumors used for this study is in progress in our laboratory.

\section{Acknowledgement}

The authors thank Tracie Dornbusch for the English editing of this article and also the 'Serena Talarico Association' for the support for this research.

\section{Disclosure/conflict of interest}

Each author warrants that he or she has no commercial associations (eg, consultancies, stock ownership, equity interest, patent/licensing arrangements, etc) that might pose a conflict of interest in connection with the work. All funding sources supporting the work and all institutional or corporate affiliations of the authors are in acknowledgements in the work.

\section{References}

1 Campanacci M, editor. Bone and Soft Tissue Tumors ed. Springer-Verlag: Wien-New York, 1999.

2 Ferrari S, Zanella L, Alberghini M, et al. Prognostic significance of immunohistochemical expression of ezrin in non-metastatic high-grade osteosarcoma. Pediatr Blood Cancer 2008;50:752-756.

3 Hunter KW. Ezrin, a key component in tumor metastasis. Trends Mol Med 2004;10:201-204. 
4 Huvos AG, editor. Bone Tumors. Diagnosis, Treatment and Prognosis ed. WB Saunders Company: Philadelphia, 1991.

5 Kansara M, Thomas DM. Molecular pathogenesis of osteosarcoma. DNA Cell Biol 2007;26:1-18.

6 Folio C, Mora MI, Zalacain M, et al. Proteomic analysis of chemonaive pediatric osteosarcomas and corresponding normal bone reveals multiple altered molecular targets. J Proteome Res 2009;8:3882-3888.

7 Leonard P, Sharp T, Henderson S, et al. Gene expression array profile of human osteosarcoma. Br J Cancer 2003;89:2284-2288.

8 Khanna C, Wan X, Bose S, et al. The membranecytoskeleton linker ezrin is necessary for osteosarcoma metastasis. Nat Med 2004;10:182-186.

9 Bretscher A, Edwards K, Fehon RG. ERM proteins and merlin: integrators at the cell cortex. Nat Rev Mol Cell Biol 2002;3:586-599.

10 McClatchey AI. Merlin and ERM proteins: unappreciated roles in cancer development? Nat Rev Cancer 2003;3:877-883.

11 Gautreau A, Poullet P, Louvard D, et al. Ezrin, a plasma membrane-microfilament linker, signals cell survival through the phosphatidylinositol 3-kinase/Akt pathway. Proc Natl Acad Sci USA 1999;96:7300-7305.

12 Martin GS. Cell signaling and cancer. Cancer Cell 2003;4:167-174.

13 Bonilha VL, Finnemann SC, Rodriguez-Boulan E. Ezrin promotes morphogenesis of apical microvilli and basal infoldings in retinal pigment epithelium. J Cell Biol 1999;147:1533-1548.

14 Dard N, Louvet-Vallee S, Santa-Maria A, et al. Phosphorylation of ezrin on threonine T567 plays a crucial role during compaction in the mouse early embryo. Dev Biol 2004;271:87-97.

15 Pujuguet P, Del Maestro L, Gautreau A, et al. Ezrin regulates E-cadherin-dependent adherens junction assembly through Rac1 activation. Mol Biol Cell 2003;14:2181-2191.

16 Grune T, Reinheckel T, North JA, et al. Ezrin turnover and cell shape changes catalyzed by proteasome in oxidatively stressed cells. FASEB J 2002;16:1602-1610.

17 Zhao H, Shiue H, Palkon S, et al. Ezrin regulates NHE3 translocation and activation after $\mathrm{Na}+$-glucose cotransport. Proc Natl Acad Sci USA 2004;101:9485-9490.

18 Tsukita S, Yonemura S. ERM (ezrin/radixin/moesin) family: from cytoskeleton to signal transduction. Curr Opin Cell Biol 1997;9:70-75.

19 Batchelor CL, Woodward AM, Crouch DH. Nuclear ERM (ezrin, radixin, moesin) proteins: regulation by cell density and nuclear import. Exp Cell Res 2004;296:208-222.
$20 \mathrm{Yu} \mathrm{Y,} \mathrm{Khan} \mathrm{J,} \mathrm{Khanna} \mathrm{C,} \mathrm{et} \mathrm{al.} \mathrm{Expression} \mathrm{profiling}$ identifies the cytoskeletal organizer ezrin and the developmental homeoprotein Six-1 as key metastatic regulators. Nat Med 2004;10:175-181.

21 Wan X, Mendoza A, Khanna C, et al. Rapamycin inhibits ezrin-mediated metastatic behavior in a murine model of osteosarcoma. Cancer Res 2005; 65:2406-2411.

22 Curto M, McClatchey AI. Ezrin ... a metastatic detERMinant? Cancer Cell 2004;5:113-114.

23 Crepaldi T, Gautreau A, Comoglio PM, et al. Ezrin is an effector of hepatocyte growth factor-mediated migration and morphogenesis in epithelial cells. J Cell Biol 1997;138:423-434.

24 Zhu L, Zhou R, Mettler S, et al. High turnover of ezrin T567 phosphorylation: conformation, activity, and cellular function. Am J Physiol Cell Physiol 2007;293:C874-C884.

25 Sato N, Funayama N, Nagafuchi A, et al. A gene family consisting of ezrin, radixin and moesin. Its specific localization at actin filament/plasma membrane association sites. J Cell Sci 1992;103(Pt 1):131-143.

26 Sarrio D, Rodríguez-Pinilla SM, Dotor A, et al. Abnormal ezrin localization is associated with clinicopathological features in invasive breast carcinomas. Breast Cancer Res Treat 2006;98:71-79.

27 Ohtani K, Sakamoto H, Rutherford T, et al. Ezrin, a membrane-cytoskeletal linking protein, is highly expressed in atypical endometrial hyperplasia and uterine endometrioid adenocarcinoma. Cancer Lett 2002;179:79-86.

28 Kaul SC, Kawai R, Nomura H, et al. Identification of a $55-\mathrm{kDa}$ ezrin-related protein that induces cytoskeletal changes and localizes to the nucleolus. Exp Cell Res 1999;250:51-61.

29 Ren L, Hong SH, Cassavaugh J, et al. The actincytoskeleton linker protein ezrin is regulated during osteosarcoma metastasis by PKC. Oncogene 2009;28:792-802.

30 Salas S, de Pinieux G, Gomez-Brouchet A, et al. Ezrin immunohistochemical expression in cartilaginous tumours: a useful tool for differential diagnosis between chondroblastic osteosarcoma and chondrosarcoma. Virchows Arch 2009;454:81-87.

31 Makitie T, Carpen O, Vaheri A, et al. Ezrin as a prognostic indicator and its relationship to tumor characteristics in uveal malignant melanoma. Invest Ophthalmol Vis Sci 2001;42:2442-2449.

32 Weng WH, Ahlen J, Astrom K, et al. Prognostic impact of immunohistochemical expression of ezrin in highly malignant soft tissue sarcomas. Clin Cancer Res 2005;11:6198-6204. 